\title{
Prueba de impulso cefálico: Bases fisiológicas y métodos de registro del reflejo vestíbulo oculomotor
}

\author{
Head impulse test: Physiological basis and registration methods of the \\ vestibulo-ocular reflex
}

\author{
Cristián Carriel P1, Mónica Rojas $\mathbf{0}^{2}$.
}

\begin{abstract}
RESUMEN
En la historia de la evaluación clínica vestibular, siempre se ha buscado la manera de medir en forma individual los seis canales semicirculares y los cuatro órganos otolíticos. Basados en el conocimiento de la fisiología vestibular, en 1988 Halmagyi y Curthoys describieron la Prueba de Impulso Cefálico o Head Impulse Test (HIT) como un método diagnóstico para evaluar la efectividad del reflejo vestíbulo oculomotor en pacientes con sintomatología vestibular.

Esta revisión pretende dar a conocer las bases fisiológicas del HIT y su evolución hasta el video HIT (vHIT) actual. bular.

Palabras claves: Head impulse test, reflejo vestíbulo oculomotor, patología vesti-

ABSTRACT

In the history of vestibular clinical evaluation, it has always been sought the way to measure individually each of the six semicircular canals and the four otolith organs. Based on vestibular physiology, in 1988 Halmagyi and Curthoys described the Head impulse test (HIT) as a diagnostic method to evaluate the effectiveness of the vestibulo-oculomotor reflex in patients with vestibular symptoms.

This review shows the physiological basis for HIT and its evolution towards nowdays video HIT (vHIT).
\end{abstract}

Key words: Head impulse test, vestibulo-ocular reflex, vestibular pathology.

\footnotetext{
${ }^{1}$ Tecnólogo Médico ORL, Servicio de Otorrinolaringología, Hospital del Salvador.

${ }^{2}$ Médico Cirujano, Universidad de Chile.
} 


\section{INTRODUCCIÓN}

En la historia de la evaluación clínica vestibular siempre se ha buscado la manera de medir en forma individual cada uno de los órganos de este sistema; los seis canales semicirculares y los cuatro órganos otolíticos en toda su gama dinámica.

Los canales semicirculares son sensibles a aceleraciones angulares. Sabemos que la frecuencia fisiológica de los movimientos cefálicos giran en torno a los $5 \mathrm{~Hz}$ en un rango de 2 a $7 \mathrm{~Hz}$ con aceleraciones de 4 a $5.000 \% \mathrm{seg}^{2}$. La prueba calórica clásica mide el funcionamiento de los canales laterales en forma individual en un rango de frecuencia ultrabajo $(0,025 \mathrm{~Hz})$, siendo considerada una prueba umbral. Por otro lado las pruebas rotacionales, como la silla rotatoria, miden la función de los canales laterales simultáneamente, pero no en forma individual y a un rango de frecuencia apenas superior a $1 \mathrm{~Hz}^{1}$. Ambas pruebas se basan en desencadenar el reflejo vestíbulo-oculomotor (RVO), pero sin medir su eficiencia.

La eficiencia del RVO la definiremos como la propiedad de este reflejo de generar movimientos oculares de fase lenta casi perfectamente compensatorios en dirección y velocidad para los movimientos de la cabeza. Esta propiedad puede ser medida y registrada mediante la Prueba del Impulso Cefálico o Head Impulse Test (HIT), no así en las pruebas calóricas y rotacionales.

Es así como basados en el conocimiento de la fisiología vestibular, desarrollado por Goldberg y Fernández en el año 1970², en 1988 Halmagyi y Curthoys describieron la Prueba del Impulso Cefálico ${ }^{3}$ como un método diagnóstico para evaluar la eficiencia del RVO.

\section{BASES FISIOLÓGICAS HIT}

Frente a un estímulo, el sistema vestibular responde de dos maneras antagónicas, estimulación e inhibición (sistema push-pul). Es así, como en un movimiento cefálico a derecha en el plano horizontal, se producirá una respuesta excitatoria en el canal semicircular lateral (CSCL) derecho y una respuesta de tipo inhibitoria en el CSCL izquierdo. No obstante, pese a ser respuestas que ocurren de forma simultánea, los estudios de Goldberg y Fer- nández mostraron que frente a movimientos de alta aceleración y corta duración, estas respuestas son desiguales entre sí al presentar tasas de descarga neuronal distintas y con diferentes magnitudes². Este fenómeno fue primeramente descrito por Ewald a fines del siglo $19^{3}$.

El HIT se basa eminentemente en esta asimetría de la respuesta del sistema canalicular a un estímulo cefálico de alta aceleración y corta duración. La asimetría se explica por la diferencia en la tasa de descarga neuronal que existe entre la excitación e inhibición de dos canales coplanares a partir de la tasa de descarga de reposo o basal. Considerando como ejemplo el CSCL derecho, frente a una rotación cefálica hacia derecha de alta aceleración, la tasa de descarga de las neuronas vestibulares aferentes primarias de dicho canal, crece en forma lineal con la estimulación, a partir de la tasa normal de reposo (90 descargas/seg. en el CSCL del mono ardilla) sin saturarse. Por el contrario, en el CSCL izquierdo, que está siendo inhibido por el estímulo cefálico, la tasa de descarga baja desde la tasa de reposo hasta las 0 descargas/seg. como máximo, sin poder inhibirse más ${ }^{4}$. Lo mismo ocurre a nivel de las neuronas secundarias a nivel del núcleo vestibular medial, donde es mucho más evidente esta diferencia entre las magnitudes de la excitación e inhibición, ya que la mayoría de estas neuronas presentan tasas de reposo aún menores que las neuronas primarias, siendo por tanto más fáciles de "silenciar" (Figura 1).

En otras palabras, si bien tanto la excitación como la inhibición del sistema canalicular son necesarias para el RVO, frente a un estímulo cefálico de alta aceleración y corta duración la mayor parte del peso en su generación recae en la excitación ${ }^{1,4}$

Considerando estas características, podría suponerse que las respuestas del RVO a movimientos cefálicos rápidos y de corta duración serían una forma de medir, en forma individual, la función de los canales semicirculares.

\section{HIT Y RVO}

El HIT consiste en movimientos pasivos e impredecibles de la cabeza de pequeña amplitud $\left(10^{\circ}-20^{\circ}\right)$, de alta velocidad $(200-400 \%$ seg) y de gran aceleración (3000-4000\%/seg ${ }^{2}$ en el plano de 


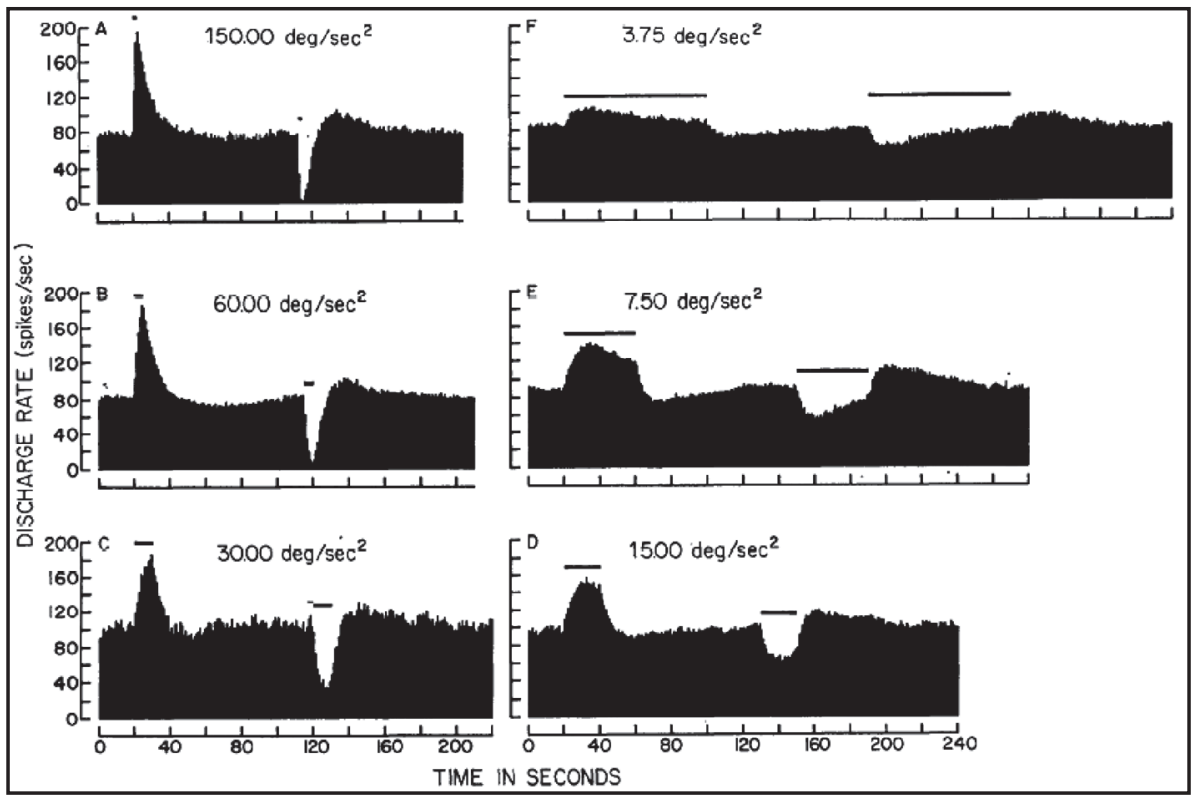

Figura 1. Goldberg y Fernández² pág. 640. Se toma como ejemplo el CSC superior. Se muestran las respuestas excitatorias e inhibitorias en presencia de aceleraciones de distintas magnitudes y duraciones. Se aprecia claramente que mientras más corto es el período de aceleración (Figura A) más evidente es la asimetría que existe entre la excitación y la inhibición, llegando esta última rápidamente a una tasa de descarga cercana al 0. Lo opuesto se observa en la Figura $\mathrm{F}$ donde a estímulos de baja aceleración y de mayor duración, la excitación e inhibición se comporta de forma más simétrica.

un par de canales semicirculares (tanto en el plano horizontal como verticales) $)^{1,5}$. En sujetos sanos, frente a una rotación cefálica, se producirá un movimiento lento de los ojos de tipo compensatorio y en dirección opuesta al movimiento de la cabeza, mediado por el RVO, para mantener la mirada fija y la imagen de interés en la fóvea ${ }^{6}$. Más fácilmente, si un sujeto fija su mirada en un punto distante y bruscamente rotamos su cabeza hacia un lado, los ojos deben ser capaces de mantener la imagen del objeto en la retina.

El HIT por tanto evalúa la función angular del laberinto, es decir los canales semicirculares, dejando de lado la función lineal que recae en los órganos otolíticos, el utrículo y el sáculo.

Cuando existe algún déficit en el RVO los ojos se moverán en la misma dirección de la rotación de la cabeza y, para mantener la mirada fija en un objeto, deberán realizar un movimiento sacádico correctivo (catch-up saccades) hacia el lado opuesto a la rotación cefálica. Si esta sacada de corrección se produce al final del movimiento cefálico, un examinador entrenado la podrá observar. Estas son conocidas como overt saccades (descubiertas) y son el signo clínico de una paresia del CSC estimulado. Sin embargo, si esta sacada correctiva aparece durante el movimiento cefálico, no podrá ser detectable a ojo desnudo. Estas sacadas son conocidas como covert saccades (encubiertas) siendo también signos de paresia vestibular. Tanto las overt saccades como las covert saccades pueden estar presentes simultáneamente en un paciente. El problema radica en que la existencia de covert saccades hace que la amplitud de las overt saccades disminuya, haciendo difícil la observación de éstas últimas a ojo desnudo y favoreciendo la aparición de falsos negativos al no registrarlas ${ }^{1,7-9}$ (Figura 2). Es así que el HIT a ojo desnudo presenta una sensibilidad entre $55 \%$ a $72 \%$ con una especificidad entre $78 \%$ y $83 \%{ }^{10}$.

Por lo anterior se han implementado diversos métodos para mejorar el registro del RVO y aumentar la sensibilidad y especificidad del HIT.

\section{REGISTRO DE LA GANANCIA DEL RVO}

El RVO es de los reflejos más rápidos en desencadenarse, aproximadamente a los 7,5 \pm 


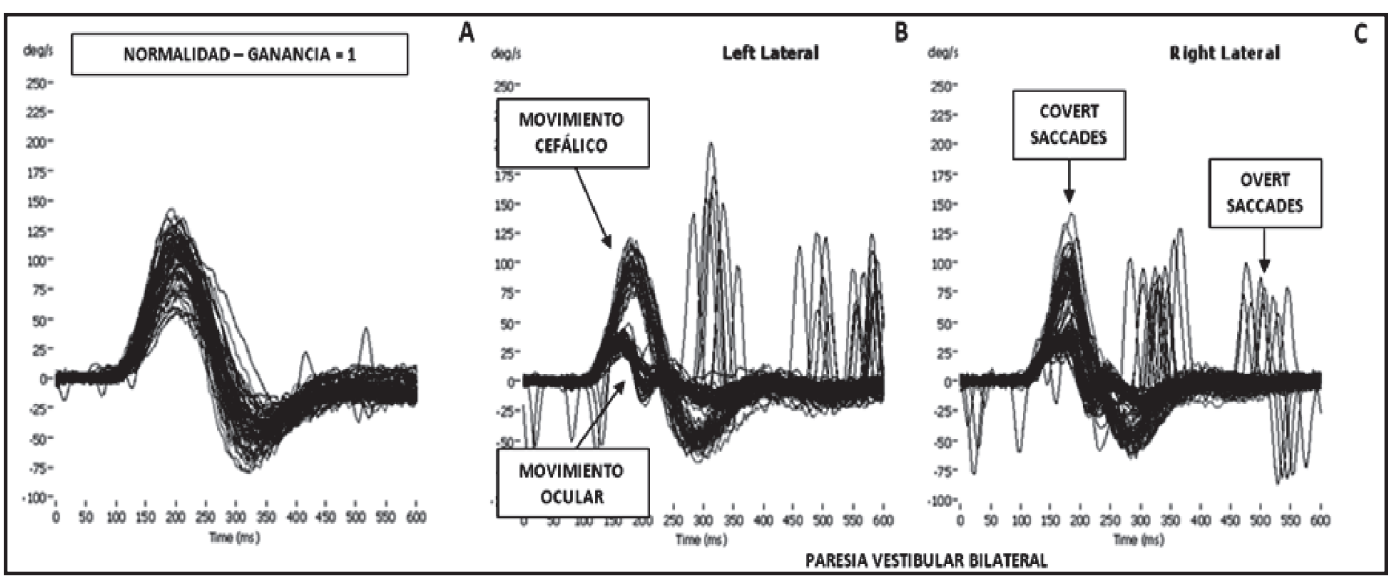

Figura 2: www.headimpulse.com ${ }^{11}$. En la Figura A se muestra el registro en un paciente normal en donde la ganancia del RV0 es igual 1, lo que significa que en la gráfica existe una superposición perfecta entre el movimiento cefálico y el movimiento ocular. Las Figuras B y C corresponde a los registros de un paciente con una paresia vestibular bilateral. En B vemos que el movimiento ocular no sigue al movimiento cefálico. En C se aprecian covert saccades durante el transcurso del impulso cefálico, por sobre el RVO residual, y overt saccades luego del movimiento cefálico durante el período de fijación ocular.

2,9 mseg. en el plano horizontal (yaw), desde el comienzo de la estimulación, casi al mismo tiempo del movimiento cefálico, por lo que es fundamental el instrumental adecuado para su registro ${ }^{5}$.

El ideal sería poder consignar la aceleración del movimiento cefálico y relacionarla con la aceleración del movimiento compensatorio ocular. Esto último se conoce como ganancia del RVO y se define como la relación que existe entre el movimiento cefálico (input) y el desplazamiento de los ojos (output). Así, la aceleración de los ojos puede ser consignada como una función de la aceleración cefálica (ganancia RVO= aceleración ocular/aceleración cefálica). En sujetos normales la ganancia del RVO tiene un valor cercano a 1, siendo en el plano horizontal de 0,94 $\pm 0,065$.

Es así como los valores de ganancia más cercanos a 010, la presencia de catch-up saccades y una ganancia asimétrica son indicativos de un RVO disminuido. La presencia de cualquiera de estos tres criterios de anormalidad es signo de paresia vestibular.

Por tanto dada sus características de ganancia y latencia, en condiciones normales el RVO genera movimientos oculares de fase lenta que son casi perfectamente compensatorios en dirección y velocidad para los movimientos de la cabeza, lo que no ocurre cuando existe una hipofunción vestibular.

\section{REGISTRO DE LA GANANCIA DEL RVO CON BOBINA ESCLERAL}

Para analizar el RVO se ha utilizando la bobina de búsqueda escleral en campo magnético (scleral search-coil). Desde su primera descripción en 1963 por Robinson ${ }^{12}$, hasta la fecha sigue siendo considerada el gold standard para el registro de los movimientos oculares. Este dispositivo consta de bobinas que generan campos magnéticos. La unidad ocular la forman 2 bobinas metálicas incluidas en un anillo de silicona, a modo de lente, que se coloca sobre la superficie escleral de manera que la pupila y el iris quedan descubiertos. Bajo el mismo principio, se usa una bobina doble montada en una impresión dental para medir los movimientos cefálicos. Ambos dispositivos deben situarse dentro de un marco cúbico mágnético de 1 metro de lado de forma que al interactuar sus campos magnéticos con los del marco es posible registrar de la posición de los ojos y de la cabeza en las tres dimensiones espaciales ${ }^{5}$ (Figura 3 ).

Mediante este método de registro la sensibilidad y especificidad aumentan respecto al HIT a ojo desnudo, siendo de $84 \%$ y $91 \%$ respectivamente ${ }^{10}$.

No obstante su utilidad, es un método caro, invasivo, molesto para el paciente y de difícil implementación. 


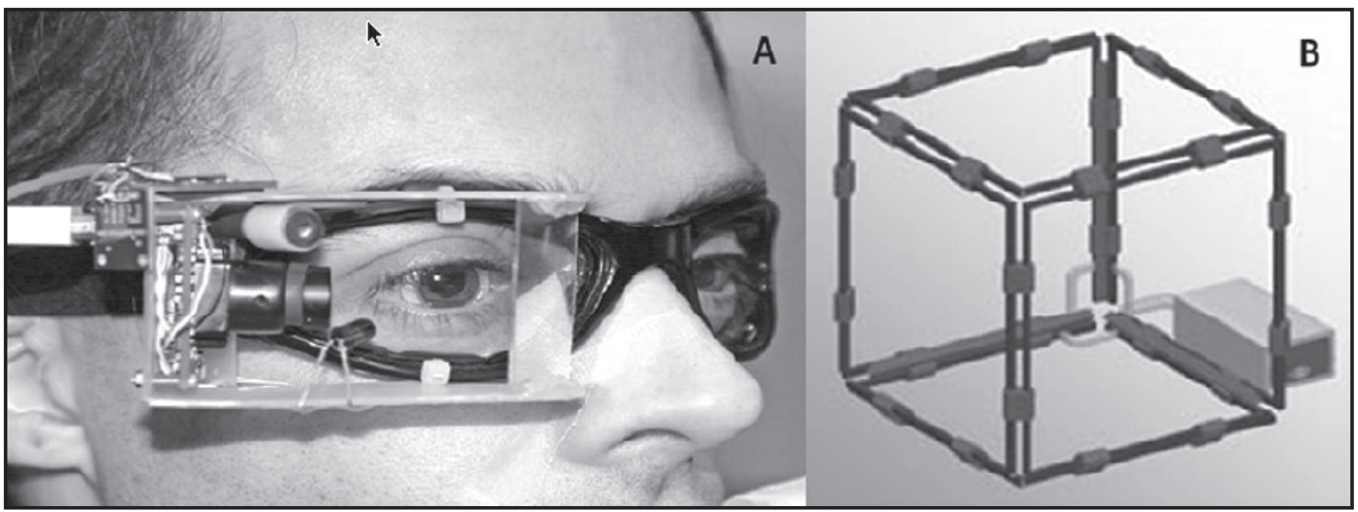

Figura 3. A: Se muestra la bobina escleral y el uso de gafas con cámaras de alta velocidad. B: Marco magnético; parte del equipamiento para el registro con bobina escleral.

\section{VIDEO HEAD IMPULSE TEST - vHIT}

Con el advenimiento de cámaras de video pequeñas, livianas y de alta velocidad, en combinación con sensores de inercia, es posible obtener los mismos registros que la bobina escleral, pero disminuyendo el costo y las molestias para el paciente.

El Video Head Impulse Test (VHIT) se basa en el registro de los movimientos oculares con el uso de cámaras de alta velocidad (250 cuadro/ segundos) que logran captar los movimientos compensatorios durante el impulso cefálico, que al ser de mayor aceleración y velocidad y de menor latencia, no son posibles de registrar con cámaras convencionales y menos a ojo desnudo.

Para el registro de los movimientos cefálicos se utiliza una unidad de medición de inercia compuesta por un acelerómetro de 3 ejes y de 2 giroscopios de doble eje que forma parte de las gafas de video-oculografía ${ }^{5}$.

Se conocen en la literatura al menos dos trabajos, uno australiano ${ }^{13}$ y otro alemán ${ }^{13}$, que comparan los registros del RVO desencadenados por impulsos cefálicos utilizando ambos métodos en forma simultánea (scleral search-coily videoculografía de alta velocidad). Con ambos sistemas se evaluaron pacientes normales y con patología vestibular conocida, registrando los movimientos oculares del ojo derecho. Para el estudio con bobina escleral los sujetos fueron sentados en el centro de un marco magnético, fijando la mirada en un objetivo colocado a una distancia de $91 \mathrm{~cm}$. aplicándoseles impulsos cefálicos aleatorios hacia uno y otro lado en el plano horizontal. Para el registro con video-oculógrafo se uso una cámara liviana, pequeña y de alta velocidad montada en un marco liviano especialmente diseñado para evitar su desplazamiento durante los movimientos de la cabeza. En sujetos normales con ambos métodos se obtuvieron ganancias del RVO cercanas a 1 en el plano horizontal'13,14 (Figura 4).

Caso 1: En un paciente con neuritis vestibular con pérdida unilateral de la función del CSCL consignada en la prueba calórica, los impulsos cefálicos hacia el lado afectado produjeron una disminución de la ganancia RVO. Este sujeto tuvo un HIT positivo presentando sacadas tipo overt, vistas a ojo desnudo, como covert registradas gracias al uso de la bobina escleral y del video-oculógrafo. Al registro ambos tipos de sacadas correctivas fueron evidenciadas y claramente distinguibles de la respuesta residual del $\mathrm{RVO}^{13}$.

Caso 2: En un paciente con vértigo, cuyo HIT a ojo desnudo fue normal sin poder evidenciarse overt saccades, pese a tener una prueba calórica casi abolida en el oído supuestamente afectado. Lo anterior estaría explicado por la presencia de 


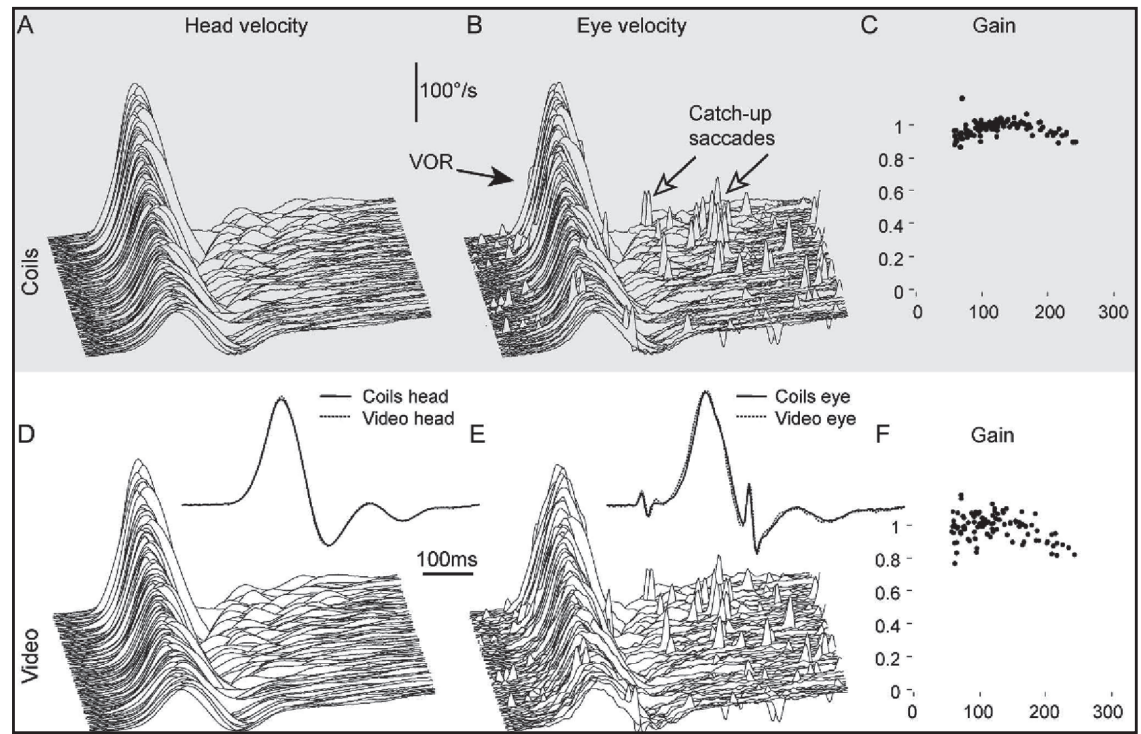

Figura 4. Mac Dougall y col$^{7}$ pág. 1136. Se observan las gráficas comparativas entre el uso de bobinas (A y B) y el uso de video cámara de alta velocidad ( $D$ y E) en un mismo paciente para la obtención de la ganancia del RVO (C y F). No se observan diferencias entre el uso de uno u otro método, tanto para los movimientos cefálicos (A y D) como para el movimiento compensatorio de los ojos (B y E). Las ganancias obtenidas al relacionar ambos registros tampoco presentan diferencias significativas.

un sinnúmero de covert saccades durante los impulsos cefálicos, que sí pudieron ser registradas al realizar el test con el uso de la bobina y videocámara. Posteriormente, con una resonancia magnética se constató la presencia de un schwannoma vestibular en el lado afectado ${ }^{13}$.

Con lo anterior no hay discusión que el registro de las sacadas correctivas, tanto overt como covert es fundamental para evitar la presencia de falsos negativos como en el segundo caso.

El uso de la bobina escleral mejora el valor diagnóstico del HIT, pero es un método caro, invasivo, molesto para el paciente y de difícil implementación. Asimismo, el vHIT ha demostrado igual efectividad en el registro de los movimientos oculares compensatorios, con una sensibilidad y especificidad cercana al $100 \%{ }^{7,10}$ y con la ventaja de ser un método fácil de implementar, de pocos minutos de duración, no invasivo, repetible en el tiempo y mejor tolerado por los pacientes.

\section{CONCLUSIÓN}

Junto con la prueba calórica, los potenciales evocados miogénicos vestibulares (VEMP), test de la visual vertical subjetiva (SVV), el registro de los movimientos oculares voluntarios y reflejos y la posturografía, el HIT con registro video-oculográfico pasa a engrosar la lista de exámenes necesarios para la correcta y completa evaluación de las patologías vestibulares. Dado que el vHIT es un procedimiento rápido, no invasivo, bien tolerado y reproducible en el tiempo por distintos examinadores entrenados, es útil para el diagnóstico y, en especial, para el seguimiento de pacientes con sintomatología vestibular.

El poder graficar los movimientos cefálicos y Ios movimientos oculares compensatorios permite un análisis de las características estímulo-respuesta del RVO, siendo posible realizar estímulos con distintos grados de magnitud y correlacionarlos con los distintos grados de respuesta, convirtiendo al VHIT en una herramienta objetiva en la evaluación de la eficiencia del RVO.

Considerando entonces que el VHIT mide la función dinámica de los canales semicirculares y, por su parte el VEMP y la SVV evalúan la función otolítica, su uso conjunto en la evaluación de un paciente con síntomas vestibulares permitiría realizar una correcta estimación de la función vestibular logrando una mejor aproximación al sitio de la lesión. 
El vHIT es un examen más dentro de los disponibles para el estudio vestibular, y al igual que ellos requiere de un análisis de sus resultados en combinación con los demás y no solo una interpretación de cada examen por separado para realizar a un diagnóstico certero.

\section{BIBLIOGRAFÍA}

1. Halmagyi G, Cremer P, Curthoys I, Todd M. Impulsive Testing of Individual Semicircular Canal Function. Ann NY Acad Sci 2001; 942 : 192-200.

2. Goldberg J, Fernández C. Physiology of Peripheal Neurons Innervating Semicircular Canals of the Squirrel Monkey. I. Resting Discharge and Response to Constant Accelerations. J Neurophysiol 1971; 34: 635-60.

3. Halmagy G, Curthoys I. A Clinical Sing of Canal Paresis. Arch Neurol 1988; 45: 737-9.

4. Wurts F. Principle of the Head Impulse (thrust) Test or Halmagyi Head Thrust Test (HHTT). B-ENT 2008; 4 Supp 8: 23-5.

5. Boleas-Aguirre M, Miglacciob A, Carey J. Registro el Reflejo Vestibulooculomotor con la Técnica de la Bobina Corneal en Campo Magnético (Scleral Search Coil). Revisión de Afecciones Vestibulares Periféricas. Acta Otorrinolaringol Esp 2007; 58(7): 321-6.

6. LeIGH R, ZeE D. The Neurology of Eye Movements. 3rd Edit. F.A. Davis Co. 1999, Philadelphia.

7. Mac Dougall H, Weber K, Mcgarvie L, Halmagyı G, Curthoys I. The Video Head Impulse Test: Diagnostic Accuracy in Peripheral Vestibulopathy. Neurology 2009; 73: 1134-41.

8. Mac Dougall H, Curthoys I. Plasticity During Vestibular Compensation: The Role of Saccades. Front Neurol 2012; 3: 21.

9. Weber K, Aw S, Todd M, Mc Garvie L, Curthoys I, Halmagy G. Head Impulse Test in Unilateral Vestibular Loss: Vestibulo-ocular Reflex and Catch-up Saccades. Neurology 2008; 70: 45463.

10. Breinbauer H, Anabalón J. Prueba de Impulso Cefálico. Rev Otorrinolaringol Cir Cabeza Cuello 2011; vol. 71, no. 2, p.123-30.

11. "Understanding vHIT data" " [en línea], [visto 7 Marzo de 2013]. Disponible en la web: www. headimpulse.com.

12. Robinson D. A Method of Measurement Eye Movements using a Scleral Search Coil in a Magnetic Field. I.E.E. E. Trans. Biomed Electron 1963; BME-10. 137-45.

13. Weber K, Mac Dougall H, Halmagy G, Curthoys I. Impulsive Testing Of Semicircular-Canal Function Using Video-Oculography. Basic and Clinical Aspects of Vertigo and Dizziness. Ann NY Acad Sci 2009; 1164: 486-91.

14. Bartl K, Lehnen N, Kohlbecher S, Schneider E. Head Impulse Testing Using Video-Oculography. Ann NY Acad Sci 2009; 1164: 331-3.

Dirección: Cristian Carriel P. 